9th A. Friedmann International Seminar and

3rd Casimir Symposium 2015

International Journal of Modern Physics: Conference Series

Vol. 41 (2016) 1660122 (5 pages)

(C) The Author(s)

DOI: $10.1142 /$ S2010194516601228

\title{
Equations of geodesic deviation and properties of tidal forces in the Kerr metric
}

\author{
A. M. Rasulova \\ Theoretical Physics and Astronomy Department, The Herzen University, Moika 48, \\ St. Petersburg, 191186, Russia \\ Institute of Limnology, RAS, Sevastyanov st. 9, \\ St. Petersburg, 196105, Russia \\ ARasulova@gmail.com \\ Received 20 September 2015 \\ Published 18 March 2016
}

\begin{abstract}
In the paper the work of the tidal forces that arise when the relative deviation of the protons on distance of the order of the Compton wavelength near the horizon of Kerr black hole is considered. For ease of calculation the assumption is made that the proton has only a radial component of the velocity. It is shown that the work of the tidal forces at speeds close to the speed of light sharply increases with Lorentz factor and it can obtain very high energy of the Grand Unification order.
\end{abstract}

Keywords: Geodesic deviation; Kerr metric; tidal forces; work of the tidal forces.

PACS numbers: 04.70.-s, 04.70.Bw, 97.60.Lf

\section{Introduction}

In a series of articles ${ }^{1-6}$ particles collisions near the horizon of rotating black holes were considered. It was shown that in center of mass frame they can achieve high energy of the Grand Unification or even Planck order. In this paper we show that the achievement of these energies near the horizon of black holes is possible due to the effect of geodesic deviation.

When driving two or more closely spaced particles in curved space-time there is a deviation of their world lines. General view of the geodesic deviation equation of the n-dimensional Riemannian manifold was obtained by T. Levi-Civita in $1925 .^{7}$ For the 4-dimensional space deviation equation for structureless massless particles was investigated in Refs. 8 and 9 by J. L. Sing.

This is an Open Access article published by World Scientific Publishing Company. It is distributed under the terms of the Creative Commons Attribution 4.0 (CC-BY) License. Further distribution of this work is permitted, provided the original work is properly cited. 


\section{A. M. Rasulova}

This effect is reduced to the geodesic deviation infinitely close to each other of world lines.

The system of units $G=c=1$ is used in the paper.

\section{The Kerr Metric}

The Kerr's metric in Boyer-Lindquist coordinates has the form: ${ }^{10}$

$$
d s^{2}=\rho^{2} \frac{\Delta}{\Sigma^{2}} d t^{2}-\frac{\Sigma^{2}}{\rho^{2}}\left[d \varphi-\frac{2 a M r}{\Sigma^{2}} d t\right]^{2} \sin ^{2} \theta-\frac{\rho^{2}}{\Delta} d r^{2}-\rho^{2} d \theta^{2},
$$

where

$$
\begin{gathered}
\Delta=r^{2}-2 M r+a^{2}, \\
\rho^{2}=r^{2}+a^{2} \cos ^{2} \theta, \\
\Sigma^{2}=\left(r^{2}+a^{2}\right)^{2}-a^{2} \Delta \sin ^{2} \theta,
\end{gathered}
$$

and $M$ is the black hole mass, $a M$ its angular momentum $0 \leq a \leq 1$. The event horizon of the Kerr's black hole corresponds to the coordinate:

$$
r_{h}=M+\sqrt{M^{2}-a^{2}} .
$$

The static limit surface is defined by the value:

$$
r_{s t}=M+\sqrt{M^{2}-a^{2} \cos ^{2} \theta} .
$$

The region of space-time between the static limit and the event horizon is called ergosphere.

In view of the equation (1) covariant and contravariant metric tensors are: ${ }^{11}$

$$
\begin{gathered}
g_{i j}=\left(\begin{array}{llll}
1-2 M r / \rho^{2} & 0 & 0 & 2 a M r \sin ^{2} \theta / \rho^{2} \\
0 & -\rho^{2} / \Delta & 0 & 0 \\
0 & 0 & -\rho^{2} & 0 \\
2 a M r \sin ^{2} \theta / \rho^{2} & 0 & 0 & -\left[\left(r^{2}+a^{2}\right)+2 a M r \sin ^{2} \theta / \rho^{2}\right] \sin ^{2} \theta
\end{array}\right), \\
g^{i j}=\left(\begin{array}{llll}
\Sigma^{2} / \rho^{2} \Delta & 0 & 0 & 2 a M r / \rho^{2} \Delta \\
0 & -\Delta / \rho^{2} & 0 & 0 \\
0 & 0 & -\frac{1}{\rho^{2}} & 0 \\
2 a M r / \rho^{2} \Delta & 0 & 0 & -\left(\Delta-a^{2} \sin ^{2} \theta\right) / \rho^{2} \Delta \sin ^{2} \theta
\end{array}\right)
\end{gathered}
$$

The nonzero components of the curvature tensor in the Kerr metric have the form: ${ }^{11}$

$$
\begin{gathered}
R_{1023}=-a M \cos \theta\left(3 r^{2}-a^{2} \cos ^{2} \theta\right) \frac{1}{\rho^{6}} \\
R_{1230}=-\frac{a M \cos \theta}{\rho^{6}}\left(3 r^{2}-a^{2} \cos ^{2} \theta\right) \Sigma^{-2}\left[\left(r^{2}+a^{2}\right)^{2}+2 a^{2} \Delta \sin ^{2} \theta\right],
\end{gathered}
$$




$$
\begin{aligned}
& R_{1302}=\frac{a M \cos \theta}{\rho^{6}}\left(3 r^{2}-a^{2} \cos ^{2} \theta\right) \Sigma^{-2}\left[2\left(r^{2}+a^{2}\right)^{2}+a^{2} \Delta \sin ^{2} \theta\right], \\
& -R_{3002}=R_{1213}=-\frac{a M \cos \theta}{\rho^{6}}\left(3 r^{2}-a^{2} \cos ^{2} \theta\right) \frac{3 a \Delta^{1 / 2}}{\Sigma^{2}}\left(r^{2}+a^{2}\right) \sin \theta, \\
& -R_{1220}=R_{1330}=-\frac{M r}{\rho^{6}}\left(r^{2}-3 a^{2} \cos ^{2} \theta\right) \frac{3 a \Delta^{1 / 2}}{\Sigma^{2}}\left(r^{2}+a^{2}\right) \sin \theta, \\
& -R_{1010}=R_{2323}=\frac{M r}{\rho^{6}}\left(r^{2}-3 a^{2} \cos ^{2} \theta\right)=R_{0202}+R_{0303}, \\
& -R_{1313}=R_{0202}=\frac{M r}{\rho^{6}}\left(r^{2}-3 a^{2} \cos ^{2} \theta\right) \Sigma^{-2}\left[2\left(r^{2}+a^{2}\right)^{2}+a^{2} \Delta \sin ^{2} \theta\right], \\
& -R_{1212}=R_{0303}=-\frac{M r}{\rho^{6}}\left(r^{2}-3 a^{2} \cos ^{2} \theta\right) \Sigma^{-2}\left[\left(r^{2}+a^{2}\right)^{2}+2 a^{2} \Delta \sin ^{2} \theta\right] .
\end{aligned}
$$

\section{Equations of Geodesic Deviation}

If there is a pair of adjacent curves $\Gamma(v)$ and $\Gamma(v+\delta v)$ (see Fig. 1), then the equation of geodesic deviation for structureless infinitely close particles has the form:

$$
\frac{d^{2} \eta^{i}}{d s^{2}}+R_{j k m}^{i} U^{j} \eta^{k} U^{m}=0
$$

where $\eta^{i}$ - infinitesimal vector deviation, $R_{j k m}^{i}=g^{i l} R_{l j k m}$ - Riemann tensor, $U^{m}$ - 4-speed.

A solution of equation (17) is a vector of deviation of world lines that covariantly describes the relative acceleration between geodesic lines.

Let us consider the equations of geodesic deviation in the Kerr metric. We find the equation of geodesic deviation for relativistic structureless particles that have

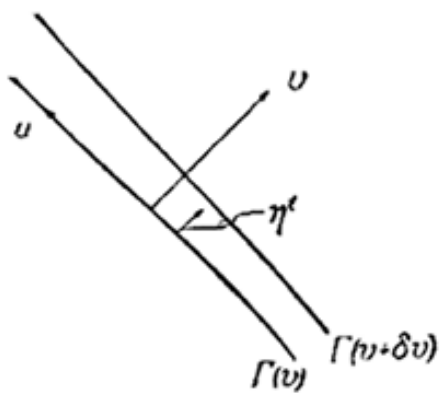

Fig. 1. The deviation of curve $\Gamma(v)$ from curve $\Gamma(v+\delta v)$. 
only radial velocity component, hence:

$$
U^{i}=\Gamma(1, V, 0,0)
$$

where $\Gamma=\frac{1}{\sqrt{1-V^{2}}}$ is Lorentz factor.

From the equations (17) and curvature tensor in the Kerr metric (9)-(16) in this case, the equation of the deviation will have the form:

$$
\begin{aligned}
\frac{D^{2} \eta^{0}}{d s^{2}}= & \Gamma^{2}\left[g_{03}\left(R_{3002}-R_{0303}\right)+g_{03} V\left(R_{1230}+R_{1330}\right.\right. \\
& \left.\left.+R_{1230}+R_{1330}\right)+V^{2}\left(g_{00} R_{1010}-g_{03} R_{1213}-g_{03} R_{1313}\right)\right], \\
\frac{D^{2} \eta^{1}}{d s^{2}}= & -g_{11} \Gamma^{2} R_{1010}(1-V), \\
\frac{D^{2} \eta^{2}}{d s^{2}}= & g_{22} \Gamma^{2}\left[R_{0202}+V\left(R_{1220}+R_{1230}\right)\right], \\
\frac{D^{2} \eta^{3}}{d s^{2}}= & \Gamma^{2}\left[g_{33}\left(R_{3002}+V^{2}\left(R_{1213}-R_{1313}\right)\right)+g_{30}\left(-R_{0303}\right.\right. \\
& \left.\left.+V^{2}\left(R_{1230}+R_{1330}-R_{1010}+R_{1302}+R_{1330}+V R_{1010}\right)\right)\right] .
\end{aligned}
$$

From these equations it is seen that the relative acceleration between the infinitely close to the world lines will be directly proportional to the square of the Lorentz factor.

To evaluate the work of the tidal forces of the proton in the Kerr metric we use the following restrictions:

- let proton with mass $m_{p}=1.67 \cdot 10^{-27} \mathrm{~kg}$ is in motion along a geodesic so that the deviation is proportional to the Compton wavelength $\lambda_{C}^{p}=1.32 \cdot 10^{-15} \mathrm{~m}$,

- let movement only occurs in the equatorial plane $\theta=\frac{\pi}{2}$,

- the black hole has the following parameters: $M=10^{6}, a=0.98$,

- motion occurs at a coordinate of distance of $r$ from horizon of black hole $r=$ $r_{h}+10^{-5} r_{h}$

- the proton velocity is $V=\left(1-10^{-15}\right) c$.

It follows from general considerations that the work of tidal forces $W$ is directly proportional to the mass of the proton $m_{p}$, the distance between the world lines $\lambda_{C}^{p}$ and the relative acceleration between geodesic $\eta^{i}$. If we consider the assumptions given above, then calculations made by us lead to the work of the tidal forces $W=21.208 \cdot 10^{30} \mathrm{eV}$.

For different values of velocities of particles a obtain by numerical calculations the work of the tidal force for protons is given in the Table 1.

The Table 1 shows how the work on the deviation of geodesics can reach high energy (of the order of Grand Unification) with increasing Lorentz factor. 
Table 1. Work of tidal forces at different speeds proton.

\begin{tabular}{ccc}
\hline $\begin{array}{c}\text { The speed of proton } V \\
(\mathrm{c})\end{array}$ & Lorentz factor $\Gamma$ & $\begin{array}{c}\text { The work of tidal forces } W \\
(\mathrm{eV})\end{array}$ \\
\hline 0.99 & 7.089 & $20.54 \cdot 10^{6}$ \\
$1-10^{-5}$ & 223.607 & $21.17 \cdot 10^{10}$ \\
$1-10^{-10}$ & $7.071 \cdot 10^{4}$ & $21.17 \cdot 10^{20}$ \\
$1-10^{-15}$ & $22.37 \cdot 10^{6}$ & $21.208 \cdot 10^{30}$ \\
\hline
\end{tabular}

\section{Acknowledgments}

The author expresses her sincere gratitude to her research supervisor Professor Andrei Grib for the problem statement and numerous discussions.

\section{References}

1. M. Bañados, J. Silk and S. M. West, Phys. Rev. Lett. 103, 111102 (2009).

2. M. Kimura, K.-i. Nakao and H. Tagoshi, Phys. Rev. D 83, 044013 (2011).

3. T. Harada and M. Kimura, Phys. Rev. D 83, 024002 (2011).

4. M. Patil and P. S. Joshi, Phys. Rev. D 84, 104001 (2011).

5. A. A. Grib, Yu. V. Pavlov and O. F. Piattella, Grav. Cosmol. 18, 70 (2012).

6. A. A. Grib and Yu. V. Pavlov, AIP Conf. Proc. 1606, 11 (2014).

7. T. Levi-Civita, The Absolute Differential Calculus (Blackie and Son, London, 1926).

8. J. L. Synge, Element Ann. Math. 35, 705 (1934).

9. J. L. Synge, Relativity: The General Theory (North-Holland, Amsterdam, 1960).

10. S. L. Shapiro and S. A. Teukolsky, Black Holes, White Dwarfs and Neutron Stars (Cornell Univ., New York, 1983).

11. S. Chandrasekhar, The Mathematical Theory of Black Holes (Oxford Univ. Press, Oxford, 1983).

12. L. D. Landau and E. M. Lifshitz, The Classical Theory of Fields (Pergamon Press, London, 1959). 